\title{
Protein Engineering Approaches for Regulating Blood-Brain Barrier Transcytosis
}

\author{
Loukas I. Goulatis and Eric V. Shusta*
}




\section{ABSTRACT}

The blood brain barrier (BBB) presents a challenge for the delivery of brain therapeutics. Trans-BBB delivery methods that use targeting vectors to coopt the vesicle trafficking machinery of BBB endothelial cells have been developed, but these are often hampered by limited flux through the BBB. A solution to this problem lies in the semi-rational engineering of BBB targeting vectors. Leveraging knowledge of intracellular trafficking, researchers have begun to tune selected binding properties of the vector-receptor interaction. Engineered binding affinity, avidity and $\mathrm{pH}$-sensitivity have been shown to affect binding, intracellular sorting and release, ultimately leading to increased brain uptake of the targeting vector and its associated cargo. However, each targeted receptor may exhibit differential responses to engineered binding properties, illustrating the need to better understand vector-receptor interactions and trafficking dynamics. 


\section{INTRODUCTION}

Therapeutic delivery for the treatment of brain diseases is hampered by the physical, transport, and metabolic properties of the blood-brain barrier (BBB). The BBB is comprised of brain microvascular endothelial cells (BMECs) that are stitched together by a network of specialized tight junctions, yielding an impressive physical barrier to blood-to-brain transport. In addition, BMECs lack fenestrae and have minimal pinocytotic activity [1]. These unique properties combine to limit the entry of blood-borne small molecule drugs and biologics into the brain [2]. However, despite its remarkable sealing properties and restriction to drug transport, the BBB is actually selectively permeable to key nutrients such as glucose, iron and lipoproteins that cross the BBB via specific molecular transport systems. This BBB phenotype may hold the solution to the brain drug delivery problem in the form of noninvasive delivery strategies that coopt such endogenous transcellular transport systems. In particular, vectors that target BBB transport systems that rely on the vesicle trafficking network can be used to deliver pharmacologically relevant doses of a variety of therapeutics [3]. Recently, the efficacy of these vectors has been improved by a combination of protein engineering approaches and a better understanding of intracellular trafficking events. In this review, we will highlight the various transcellular BBB pathways available for noninvasive brain drug delivery and the protein engineering efforts that have been deployed to best utilize the available pathway capacity. 


\section{Trans-BBB brain delivery pathways}

Transport mechanisms employing the vesicle-mediated trafficking systems of BMECs can be used to traffic drug cargo into and across the BBB. Several classes of vesiclemediated transport systems function at the BBB including fluid phase endocytosis, absorptive-mediated endocytosis, and receptor-mediated transport in the form of caveolae- or clathrin- mediated mechanisms [4,5] (Figure 1). In the case of employing receptor-mediated systems for drug delivery, an antibody or peptide is usually employed as a targeting vector. A therapeutic can either be attached directly to the targeting vector or loaded into liposomes or nanoparticles (NPs), themselves coated with the targeting vector [6]. After binding and endocytosing into BMECs by one of the aforementioned mechanisms, the vector and attached therapeutic cargo is then trafficked within the endothelial cell, with some cargo ultimately being released into the brain parenchyma by transcytosis (Figure 1). Interactions between the targeting vector and the respective transcytosis pathway components are key to determining where the therapeutic traffics and to what extent it may transcytose as discussed in the specific examples below. In this way, therapeutics can enter the central nervous system (CNS) noninvasively from the bloodstream. Below, we break down the various vesiclemediated transcytosis pathways as they have been employed in BBB drug delivery.

\section{Fluid phase transcytosis}

Fluid phase endocytosis serves as a non-specific uptake method allowing cells to internalize extracellular fluid. Endocytosis follows actin cytoskeletal rearrangements that result in formation of membrane ruffles that occasionally fold back on themselves and envelop fluid domains [7]. The resulting vesicles are devoid of coating material required 
for the curvature of smaller vesicles, distinguishing them as pinocytotic vesicles that can ultimately transcytose and release contents into the brain parenchyma [7] (Figure 1). While fluid phase endocytosis is capable of delivering payload across the BBB, challenges include its inherently nonspecific nature, inability to concentrate the therapeutic since it involves engulfment of fluid at the prevailing blood concentration, and the difficulty of tuning the transcytosis process. Moreover, one of the unique features of the BBB is that it possesses comparatively few pinocytotic vesicles [8] and therefore, the total trans-BBB flux of therapeutic by this route will be limited. Therefore, much of the trans-BBB delivery focus has been on targetable transport routes.

\section{Absorptive-mediated transcytosis}

One approach to add a first level of targeting capability is surface modification of the therapeutic carrier or the therapeutic itself through cationization. AMT is initiated by the absorption of cationic molecules to negatively charged moieties present in the surface of the brain endothelia (e.g. sialo-glycoconjugates and heparan sulfate proteoglycans) [9] (Figure 1). Using the model AMT substrate, wheat germ agglutinin, it has been shown that following internalization, cargo is directed towards the endo-lysosomal pathway, with in vivo studies showing accumulation also in the inner saccules of the golgi network, a destination not accessible to fluid phase endocytosed cargo [10]. Protein cationization by carboxyl group modification has been performed for albumin and antibodies in order to enhance the delivery of pharmaceutical payloads to the brain [11]. For instance, cationized Immunoglobulin $(\lg G)$ and cationized bovine albumin transcytosed into brain following carotid arterial infusion [12]. Further, NPs coated with cationic moieties [13] and cell penetrating peptides (CPPs) such as SynB vectors [14] 
have been employed to deliver functionalized small molecules, proteins, and nanoparticles across the BBB by AMT. Though AMT allows trans-BBB delivery of drugs in concentrated transcytotic vesicles, it remains a non-specific approach as the therapeutic will also accumulate in the blood vessels of peripheral organs leading to potential off-target effects. Moreover, the cationization process has the potential to affect the therapeutic activity. Thus, other approaches that both allow drug concentration and employ targeting of specific BBB receptors have been developed.

\section{Clathrin-mediated transcytosis}

Upon ligand and receptor engagement, clathrin-mediated transcytosis (CMT) starts with the formation of clathrin-coated pits which are then endocytosed and fused with the early, or sorting endosome (EE) (Figure 1). The EE is the main sorting compartment in the endocytic pathway and is the acceptor vesicle for other major endocytosis pathways besides CMT [15]. Membrane cargoes are sorted within the complex EE structure that involves so-called tubular and vacuolar domains. Immediately after the formation of clathrin-coated vesicles, acidification driven by vacuolar $\mathrm{H}^{+}$-ATPases begins and continues through the endosome-lysosome maturation process. Of particular relevance to CMT-targeting, $\mathrm{pH}$-induced dissociation of ligands from their cognate CMT receptors starts in the EE conditions of $\mathrm{pH} 6.8-6.1$, thereby providing a sorting mechanism separating bound ligand from the fluid phase (Figure 1). Cargoes are then spatially sequestered and sorted [16]. The main spherical vacuole matures further to become the late endosome, and finally the acidic $\mathrm{pH} 4.5$ lysosome where any accompanying material is destined for degradation. In contrast, cargo that resides in the tubular domains can either be recycled, directed to different intracellular sites or ultimately 
transcytosed in polarized cells such as BBB endothelium (Figure 1). As will be discussed below, the relative balance between these various pathways is key to maximizing the brain uptake of CMT-targeted therapeutics.

Among the most widely used CMT systems are the transferrin receptor (TfR) [17], the insulin receptor [18], and low-density lipoprotein receptor-related protein receptor $1 / 2$ (LRP) [6] all of which are highly expressed on human brain microvasculature. Another receptor believed to involve CMT processes at the BBB is the cell surface a(2,3)sialoglycoprotein TMEM-30A that has been targeted by a single domain antibody (sdAb) known as FC5 $[19,20]$. CMT offers the advantage of selective targeting compared with AMT, although the aforementioned CMT receptors, while abundant at the BBB, are expressed in peripheral endothelia as well. Finally, although CMT can direct transcellular movement of targeted therapeutics, the relative distribution of the various sorting pathways is not necessarily directed towards maximum transcytosis. Thus, engineering strategies that attempt to address this issue have been developed.

\section{Caveolae-mediated transcytosis}

Caveolae are flask-shaped membrane invaginations that are structurally and functionally different than clathrin-coated endocytotic structures (Figure 1). These primary endocytic vesicles are distinct from intracellular compartments found in the clathrin pathway, are $\mathrm{pH}$ neutral, and devoid of usual clathrin internalized ligands such as transferrin [21]. Caveolae-mediated transcytosis has been implicated in brain delivery of a number of virus derived peptides [22,23], toxins [24] and NPs [25] and has been suggested to be involved in the trafficking of the LDL receptor [26] and the $A \beta$ peptide via the receptor for advanced glycation end-products (RAGE) [27]. 
Considerable research has been performed to identify BBB-resident clathrin- or caveolae-mediated transcytosis systems and peptide or antibody vectors capable of selectively targeting them [28]. For example, a recent proteomic evaluation revealed the highly expressed CD98 heavy chain (CD98hc) as a new target for BBB delivery, and this receptor exhbited colocalization with caveolin-1 [29].

\section{Engineering BBB-targeting vectors}

Key to the efficient implementation of BBB targeting vectors are protein engineering approaches that improve trans-BBB transport. Binding properties of targeting vectors have been engineered for enhanced trans-BBB trafficking and the decoration of NPs with targeting vectors has also been explored for improved targeting and trans-BBB transport (Figure 2).

\section{Engineering targeting vectors for enhanced trans-BBB trafficking}

The amount of peptide- or antibody-targeted therapeutic that not only endoctyoses, but also transcytoses the BBB, can be limited by the intrinsic vesicle trafficking process. Binding on the blood side, endocytosis, sorting, exocytosis and brain side release are all key components in regulating the fraction of the blood-borne dose that transits the BBB and enters the parenchyma [6] (Figure 2). Recent efforts have leveraged antibody engineering strategies to increase trans-BBB transport and have highlighted the importance of the vector binding and trafficking issues. The most well-developed set of examples deals with engineering the binding properties of anti-TfR antibodies and exploring their effects on trafficking and delivery in vitro and in vivo. 
First, antibody affinity and avidity for TfR was evaluated, and it was demonstrated that higher brain uptake of anti-TfR antibodies can be accomplished by lowering antibody affinity [30]. Intravenous administration of antibodies having a range of affinity to TfR $(\mathrm{Kd}=6.9-111 \mathrm{nM})$ indicated that at trace doses, mouse brain uptake directly correlated with affinity suggesting that receptor engagement at the blood side of the BBB was the key parameter governing uptake (Figure 2). However, at therapeutic dosing $(20 \mathrm{mg} / \mathrm{kg})$, an inverse correlation was observed where the lowered affinity antibody demonstrated greater brain accumulation (up to $0.6 \% \mathrm{ID} / \mathrm{g}$ ). In addition, immunohistochemistry revealed more substantial colocalization of low affinity anti-TfR antibodies with neuronal markers as opposed to high affinity antibody clones that showed predominantly vascular localization. These data were posited to demonstrate that lowered affinity allows for antibody release from the TfR at the abluminal membrane while higher affinity variants remain bound to the TfR. Further, reduction of cortical TfR levels after administration of high affinity anti-TfR antibody was observed and mechanistic studies suggested it to be a result of increased lysosomal sorting and degradation of the high affinity variant within BMECs [31]. Thus, productive trans-BBB anti-TfR antibody trafficking could be increased by lowering antibody affinity. In addition to studies of TfR antibody binding affinity, the role of avidity has also been explored with similar conclusions. In order to investigate avidity effects on trans-BBB transport, an Fab fragment targeting the TfR was fused to the carboxy-terminus of an anti-BACE1 antibody in a bivalent (dFab) or monovalent (sFab) format [32]. The dFab exhibited a higher degree of colocalization with lysosomes compared to the sFab in the murine b.End3 BBB cell line (Figure 2). Moreover, dFab transcytosis was not detected across 
the human hCMEC/D3 BBB cell line whereas the sFab was found to transcytose. In an in vivo setting, the dFab was unable to substantially cross the mouse BBB and could be found colocalized with LAMP2, a lysosomal marker. In a mouse model of Alzheimer's disease, decreases in amyloid plaques were only observed after sFab administration, with the dFab remaining largely sequestered in the microvessels [32]. Receptor crosslinking has been associated with altered TfR trafficking [33,34], and this phenomenon likely plays an important role in BBB sequestration and degradation of high affinity and bivalent anti-TfR antibodies and their coordinate decreases in brain uptake.

In addition to modulating targeting vector binding affinity or avidity, one could also envision regulating vector-receptor trafficking dynamics by engineering binding properties that are responsive to the trafficking environment. For instance, during CMT, acidification is a key step in the vesicle maturation and sorting process [16] (Figure 1), and this $\mathrm{pH}$ gradient has been exploited in protein engineering involving FcRn binding, as well as in natural ligands including transferrin [35]. Along these lines, early work has investigated whether or not $\mathrm{pH}$-sensitive binding could be an additional engineering handle for regulating anti-TfR antibody trafficking and increasing trans-BBB transport [36]. Using in vitro BBB models, differential intracellular trafficking was observed for a $\mathrm{pH}$-insensitive anti-TfR antibody, 128.1, compared to a $\mathrm{pH}$-sensitive anti-TfR antibody, MEM-189. Antibody 128.1 colocalized with the late endosomal/lysosomal marker CD63 while MEM-189 was instead found in vesicular structures distinct from late endosomes. Transcytosis of $\mathrm{pH}$-sensitive MEM-189 across the hCMEC/D3 cell line increased; and in contrast, the lysosomal degradation of 128.1 was increased (Figure 2). These data are 
suggestive of $\mathrm{pH}$-sensitive binding being a candidate for increasing trans-BBB transport, although the epitopes for these two antibodies may not be identical. In other recent studies, $\mathrm{pH}$-sensitivity has been engineered into an anti-TfR antibody using histidine scanning mutagenesis methods, such that the resultant antibodies presumably recognize the same epitope as the $\mathrm{pH}$-insensitive wild-type antibody [37]. As in the study with 128.1 and MEM-189, antibody trafficking in SK-BR-3 cells was also substantially affected by $\mathrm{pH}$-sensitivity, with increased intracellular accumulation in distinct vesicular structures that did not appear endosomal or lysosomal in origin. These combined data are suggestive that engineered $\mathrm{pH}$-sensitive binding to the $\mathrm{TfR}$ is a viable target for altering intracellular trafficking and ultimately enhancing trans-BBB transport.

It is important to note that not all trafficking pathways will likely respond to antibody binding properties in the same fashion as the TfR pathway. For example, in a recent publication, antibodies having different affinity against basigin and CD98hc were evaluated regarding their transcytosis capability following trace and therapeutic dosing in mice [29]. While the high affinity basigin antibody exhibited increased uptake upon trace dosing like that observed with the high affinity anti-TfR antibody [29], both basigin antibodies (13.5 - 102nM affinity range) showed similar brain penetration at therapeutic dosing, which contrasts with anti-TfR antibody affinity results $[30,31,38]$. When produced as bispecific antibody constructs with an anti-BACE1 arm, anti-CD98hc/ BACE1 bispecific antibodies exhibited a similar inverse relation between antibody affinity and brain exposure to that previously observed with anti-TfR/BACE1 antibodies [38]. However, unlike anti-TfR antibodies, the high and low affinity anti-CD98hc/BACE1 
antibodies did not perturb trafficking or exhibit differential lysosomal uptake [29]. As another example, the effects of avidity on the trans-BBB passage of the FC5 sdAb mentioned above were investigated. FC5 was fused either in monovalent or bivalent format to human Fc region. In contrast to the TfR system, increased transcytosis of the bivalent construct (80\% increase) was observed using an in vitro BBB model [39] (Figure 2). Despite the differences observed in vitro, levels of the monovalent and bivalent FC5 in the cerebrospinal fluid were not statistically different. Taken together, these results indicate that optimization of antibody properties needs to be tailored to each targeted trafficking receptor, and that antibodies targeting different receptors or even different epitopes of the same receptor may have different optimal binding properties.

Finally, in addition to enhancing trans-BBB passage, factors such as immunogenicity, clearance, peripheral uptake, off-target toxicity and safety can be addressed through antibody engineering strategies. As one example, the safety liabilities of an anti-TfR antibody were mitigated by using a lowered affinity antibody and an aglycosylated Fc region that lacks effector function [40]. As another example, a murine anti-human insulin receptor antibody 83-14 has been shown to accumulate in rhesus monkey brain after intravenous administration [41]. A humanized version of the antibody was developed (HIRMAB) to lower any potential immunogenicity and despite a $27 \%$ reduction in affinity, the antibody still bound to primate and human insulin receptor and accumulated in rhesus monkey brain [42]. 


\section{Engineering NPs for increased brain penetration}

NPs have been employed to deliver pharmaceutical payloads to the CNS [43]. In vitro studies regarding the transcytosis mechanism of non-functionalized NPs have found that NPs cross brain endothelial cells by a combination of fluid phase transcytosis [44], clathrin-mediated transcytosis and caveolae-mediated transcytosis [45], albeit at rather low efficiency. Therefore, coating NPs with vectors capable of targeting specific endocytosis pathways has been shown to influence their intracellular fate, as well as their transcytosing capacity (Figure 2). For example, NP trafficking in vitro was altered by coating NPs with polyethyleneimine (PEI) or prion protein. PEI-coated NPs entered the cell via macropinocytosis and exhibited diminished transcytosis while prion-coated NPs targeted receptor-mediated systems and exhibited enhanced transcytosis compared with uncoated NPs [46]. Multiple forms of NP functionalization can synergize to further increase cellular uptake, and transcytosis capability by leveraging multiple entry mechanisms. For instance NPs synthesized from AMT-targeting cationic PAMAM polymer and decorated with CMT-targeting lactoferrin (Lf) exhibited increased brain endothelial cell uptake than non-targeted PAMAM NPs by employing both AMT and CMT mechanisms [47]. Finally, similar to antibody-based vectors, engineering the binding properties of the NP can influence transcytosis. For example, tuning the avidity of transferrin-decorated gold NPs regulated their brain accumulation in mice [48]. NPs with the highest avidity to the TfR were sequestered at the brain vasculature and exhibited reduced accumulation in the brain relative to NPs with low avidity, similar to observations of anti-TfR antibody affinity and avidity described above. Although not directly related to engineering the targeting moiety, NP shape and size can also 
influence BBB trafficking. For instance, a study employing the hCMEC/D3 cell line and electron microscopy found that $\mathrm{SiO}_{2}$ NPs of sizes ranging from $50-200 \mathrm{~nm}$ all internalized into the endo-lysosomal pathway, with the smaller NPs accumulating to a greater extent [44]. Moreover, after intravenous administration of anti-transferrin receptor-targeted polystyrene NPs, rod-shaped, rather than spherical, NPs were found to better accumulate in all murine tissues, including brain, likely as a combination of blood flow and NP adhesion effects [49].

\section{Conclusions}

Despite the obstacle presented by the BBB, the delivery of therapeutics employing endogenous BBB transcytosis mechanisms is moving forward. Targeting vector engineering has been successfully deployed to increase the amount of trans-BBB transport. Moving forward, it is anticipated that the simultaneous targeting of multiple pathways will be used to enhance uptake and specificity. In addition, one could envision employing sequential targeting of multiple pathways or environment-dependent binding to more substantially influence transcellular trafficking (e.g. cell surface targeting followed by intracellular redirection). Finally, it is our opinion that the mechanistic aspects of transcellular BBB trafficking should be an expanding research focus to better inform BBB targeting vector design and optimization.

\section{Acknowledgments}

This work was supported by Defense Threat Reduction Agency grant HDTRA1-15-1-0012. 


\section{References:}

1. Hawkins BT, Davis TP: The Blood-Brain Barrier / Neurovascular Unit in Health and Disease. Pharmacol. Rev. 2005, 57:173-185.

2. Pardridge WM: Blood-brain barrier delivery. Drug Discov. Today 2007, 12:54-61.

3. Pardridge WM: Molecular Trojan horses for blood-brain barrier drug delivery. Curr. Opin. Pharmacol. 2006, 6:494-500.

4. Jones AR, Shusta E V: Blood-brain barrier transport of therapeutics via receptormediation. Pharm. Res. 2007, 24:1759-71.

5. Smith MW, Gumbleton M: Endocytosis at the blood-brain barrier: from basic understanding to drug delivery strategies. J. Drug Target. 2006, 14:191-214.

6. Lajoie JM, Shusta E V.: Targeting Receptor-Mediated Transport for Delivery of Biologics Across the Blood-Brain Barrier. Annu. Rev. Pharmacol. Toxicol. 2015, $55: 613-631$.

7. Lim JP, Gleeson P a: Macropinocytosis: an endocytic pathway for internalising large gulps. Immunol. Cell Biol. 2011, 89:836-843.

8. Brightman MW, Reese TS: JUNCTIONS BETWEEN INTIMATELY APPOSED CELL MEMBRANES IN THE VERTEBRATE BRAIN. J. Cell Biol. 1969, 40:648-677.

9. Hervé F, Ghinea N, Scherrmann J-M: CNS delivery via adsorptive transcytosis.. AAPS J. 2008, 10:455-72.

10. Broadwell RD, Balin BJ, Salcman M: Transcytotic pathway for blood-borne protein through the blood-brain barrier. Proc. Natl. Acad. Sci. U. S. A. 1988, 85:632-636.

11. Bickel U, Yoshikawa T, Pardridge WM: Delivery of peptides and proteins through the blood-brain barrier. Adv. Drug Deliv. Rev. 2001, 46:247-279. 
12. Triguero D, Buciak J, Pardridge WM: Capillary depletion method for quantification of blood-brain barrier transport of circulating peptides and plasma proteins. $J$. Neurochem. 1990, 54:1882-1888.

13. Béduneau A, Saulnier P, Benoit JP: Active targeting of brain tumors using nanocarriers. Biomaterials 2007, 28:4947-4967.

14. Drin G, Rousselle C, Scherrmann J-M, Rees AR, Temsamani J: Peptide delivery to the brain via adsorptive-mediated endocytosis: advances with SynB vectors. AAPS PharmSci 2002, 4:E26.

15. Mayor S, Pagano RE: Pathways of clathrin-independent endocytosis. Nat. Rev. Mol. Cell Biol. 2007, 8:603-612.

16. Huotari J, Helenius A: Endosome maturation. [Internet]. EMBO J. 2011, 30:3481-3500.

17. Wilfred A. Jefferies, Malcolm R. Brandon, Simon V. Hunt, Alan F. Williams, Kevin C. Gatter DYM: Transferrin receptor on endothelium of brain capillaries. Nature 1984, 312:162-163.

18. Duffy KR, Pardridge WM: Blood-brain barrier transcytosis of insulin in developing rabbits. Brain Res. 1987, 420:32-38.

19. Haqqani AS, Delaney CE, Tremblay T-L, Sodja C, Sandhu JK, Stanimirovic DB: Method for isolation and molecular characterization of extracellular microvesicles released from brain endothelial cells. Fluids Barriers CNS 2013, 10:4.

20. Muruganandam A, Tanha J, Narang S, Stanimirovic D, Aims S: Selection of phagedisplayed Ilama single-domain antibodies that transmigrate across human blood brain barrier endothelium 1. FASEB J. 2002, 16:240-242.

21. Pelkmans L, Helenius A: Endocytosis via caveolae. Traffic 2002, 3:311-320.

22. Liu Y, Huang R, Han L, Ke W, Shao K, Ye L, Lou J, Jiang C: Brain-targeting gene 
delivery and cellular internalization mechanisms for modified rabies virus glycoprotein RVG29 nanoparticles. Biomaterials 2009, 30:4195-4202.

23. Park T-E, Singh B, Li H, Lee J-Y, Kang S-K, Choi Y-J, Cho C-S: Enhanced BBB permeability of osmotically active poly(mannitol-co-PEI) modified with rabies virus glycoprotein via selective stimulation of caveolar endocytosis for RNAi therapeutics in Alzheimer's disease. Biomaterials 2015, 38:61-71.

24. Wang P, Xue Y, Shang X, Liu Y: Diphtheria toxin mutant CRM197-mediated transcytosis across blood-brain barrier in vitro. Cell. Mol. Neurobiol. 2010, 30:717725.

25. Gao H, Yang Z, Zhang S, Pang Z, Jiang X: Internalization and subcellular fate of aptamer and peptide dual-functioned nanoparticles. J Drug Target. 2014, 2330:1-10.

26. Candela P, Gosselet F, Miller F, Buee-Scherrer V, Torpier G, Cecchelli R, Fenart L: Physiological pathway for low-density lipoproteins across the blood-brain barrier: transcytosis through brain capillary endothelial cells in vitro. Endothelium 2008, 15:254-264.

27. Candela P, Gosselet F, Saint-Pol J, Sevin E, Boucau MC, Boulanger E, Cecchelli R, Fenart L: Apical-to-basolateral transport of amyloid- $\beta$ peptides through blood-brain barrier cells is mediated by the receptor for advanced glycation end-products and is restricted by p-glycoprotein. J. Alzheimer's Dis. 2010, 22:849-859.

28. Stutz C, Zhang X, Shusta E: Combinatorial approaches for the identification of brain drug delivery targets. Curr. Pharm. Des. 2014, 20:1564-1576.

29. Zuchero YJY, Chen X, Bien-Ly N, Bumbaca D, Tong RK, Gao X, Zhang S, Hoyte K, Luk W, Huntley MA, et al.: Discovery of Novel Blood-Brain Barrier Targets to Enhance Brain Uptake of Therapeutic Antibodies. Neuron 2016, 89:70-82.

30. Yu YJ, Zhang Y, Kenrick M, Hoyte K, Luk W, Lu Y, Atwal J, Elliott JM, Prabhu S, Watts RJ, 
et al.: Boosting brain uptake of a therapeutic antibody by reducing its affinity for a transcytosis target. Sci. Transl. Med. 2011, 3:84ra44.

31. Bien-Ly N, Yu YJ, Bumbaca D, Elstrott J, Boswell CA, Zhang Y, Luk W, Lu Y, Dennis MS, Weimer RM, et al.: Transferrin receptor (TfR) trafficking determines brain uptake of TfR antibody affinity variants. J. Exp. Med. 2014, 211:233-44.

32. Niewoehner J, Bohrmann B, Collin L, Urich E, Sade H, Maier P, Rueger P, Stracke JO, Lau W, Tissot AC, et al.: Increased brain penetration and potency of a therapeutic antibody using a monovalent molecular shuttle. Neuron 2014, 81:49-60.

33. Weflen AW, Baier N, Tang Q-J, Van den Hof M, Blumberg RS, Lencer WI, Massol RH: Multivalent immune complexes divert FcRn to lysosomes by exclusion from recycling sorting tubules. Mol. Biol. Cell 2013, 24:2398-405.

34. Marsh EW, Leopold PL, Jones NL, Maxfield FR: Oligomerized transferrin receptors are selectively retained by a lumenal sorting signal in a long-lived endocytic recycling compartment. J. Cell Biol. 1995, 129:1509-1522.

35. Lao BJ, Kamei DT: Improving therapeutic properties of protein drugs through alteration of intracellular trafficking pathways. Biotechnol. Prog. 2008, 24:2-7.

36. Sade H, Baumgartner C, Hugenmatter A, Moessner E, Freskgård P-O, Niewoehner J: A Human Blood-Brain Barrier Transcytosis Assay Reveals Antibody Transcytosis Influenced by pH-Dependent Receptor Binding. [Internet]. PLoS One 2014, 9:e96340.

37. Tillotson BJ, Goulatis LI, Parenti I, Duxbury E, Shusta E V.: Engineering an antitransferrin receptor ScFv for $\mathrm{pH}$-sensitive binding leads to increased intracellular accumulation. PLoS One 2015, 10:1-21.

38. Couch J a., Yu YJ, Zhang Y, Tarrant JM, Fuji RN, Meilandt WJ, Solanoy H, Tong RK, Hoyte K, Luk W, et al.: Addressing Safety Liabilities of TfR Bispecific Antibodies 
That Cross the Blood-Brain Barrier. Sci. Transl. Med. 2013, 5:183ra57-183ra57.

39. Farrington GK, Caram-Salas N, Haqqani AS, Brunette E, Eldredge J, Pepinsky B, Antognetti G, Baumann E, Ding W, Garber E, et al.: A novel platform for engineering blood-brain barrier-crossing bispecific biologics. FASEB J. 2014, 28:4764-4778.

40. Couch J a, Yu YJ, Zhang Y, Tarrant JM, Fuji RN, Meilandt WJ, Solanoy H, Tong RK, Hoyte K, Luk W, et al.: Addressing safety liabilities of TfR bispecific antibodies that cross the blood-brain barrier. Sci. Transl. Med. 2013, 5:183ra57, 1-12.

41. Pardridge WM, Kang YS, Buciak JL, Yang J: Human insulin receptor monoclonal antibody undergoes high affinity binding to human brain capillaries in vitro and rapid transcytosis through the blood-brain barrier in vivo in the primate. Pharm. Res. 1995, 12:807-816.

42. Boado RJ, Zhang Y, Zhang Y, Pardridge WM: Humanization of anti-human insulin receptor antibody for drug targeting across the human blood-brain barrier. Biotechnol. Bioeng. 2007, 96:381-391.

43. Kreuter J: Drug delivery to the central nervous system by polymeric nanoparticles: What do we know?. Adv. Drug Deliv. Rev. 2014, 71:2-14.

44. Ye D, Raghnaill MN, Bramini M, Mahon E, Åberg C, Salvati A, Dawson K a: Nanoparticle accumulation and transcytosis in brain endothelial cell layers. Nanoscale 2013, 5:11153-65.

45. Ye D, Anguissola S, O'Neill T, Dawson K a.: Immunogold labeling reveals subcellular localisation of silica nanoparticles in a human blood-brain barrier model. Nanoscale 2015, 7: 10050-8.

46. Georgieva J V, Kalicharan D, Couraud P-O, Romero I a, Weksler B, Hoekstra D, Zuhorn IS: Surface characteristics of nanoparticles determine their intracellular fate in and processing by human blood-brain barrier endothelial cells in vitro. Mol. Ther. 2011, 
19:318-325.

47. Huang R, Ke W, Han L, Liu Y, Shao K, Ye L, Lou J, Jiang C, Pei Y: Brain-targeting mechanisms of lactoferrin-modified DNA-loaded nanoparticles. J. Cereb. Blood Flow Metab. 2009, 29:1914-1923.

48. Wiley DT, Webster P, Gale A, Davis ME: Transcytosis and brain uptake of transferrincontaining nanoparticles by tuning avidity to transferrin receptor. Proc. Natl. Acad. Sci. U. S. A. 2013, 110:8662-7.

49. Kolhar P, Anselmo AC, Gupta V, Pant K, Prabhakarpandian B, Ruoslahti E, Mitragotri S: Using shape effects to target antibody-coated nanoparticles to lung and brain endothelium. Proc. Natl. Acad. Sci. U. S. A. 2013, 110:10753-8.

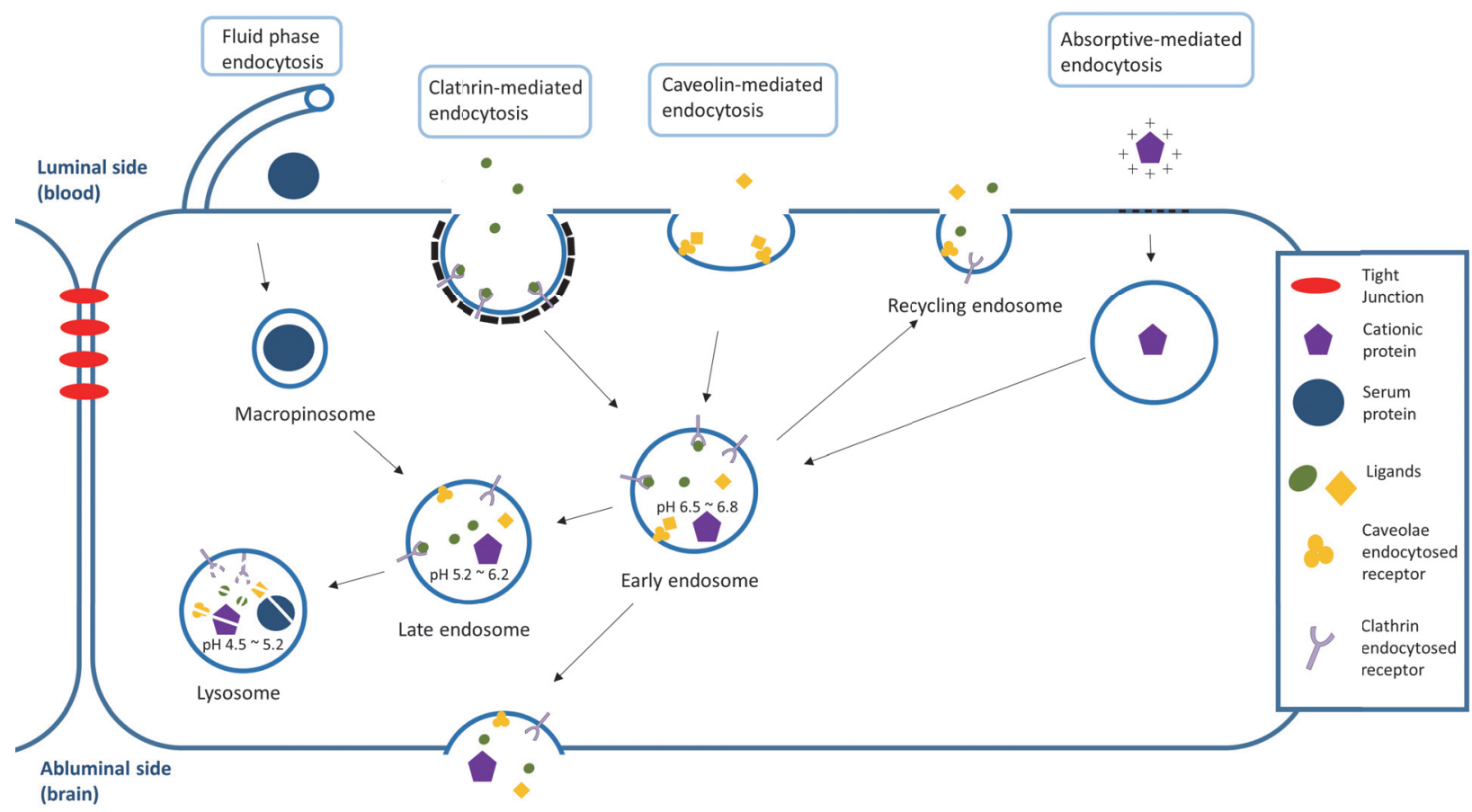

Figure 1. Endogenous BBB endocytosis and transcytosis pathways. Fluid phase endocytosis involves the generation of large vesicles that internalize extracellular fluid and soluble proteins. Absorptive-mediated endocytosis is initiated by the nonspecific 
absorption of cationic molecules to the negatively charged plasma membrane and subsequent trafficking through the endosome towards either degradation, or transcytosis. Clathrin-mediated endocytosis involves the formation of clathrin and adaptor protein complexes that result in the formation of a clathrin-coated vesicles. These vesicles mature to EEs, where cargo can then be sorted and trafficked for recycling, degradation or transcytosis. Caveolae-mediated endocytosis begins with the formation of vesicles that are structurally and functionally different from clathrin-coated vesicles, followed by delivery of the receptor-ligand complex to the EE where it can be further processed for transcytosis.

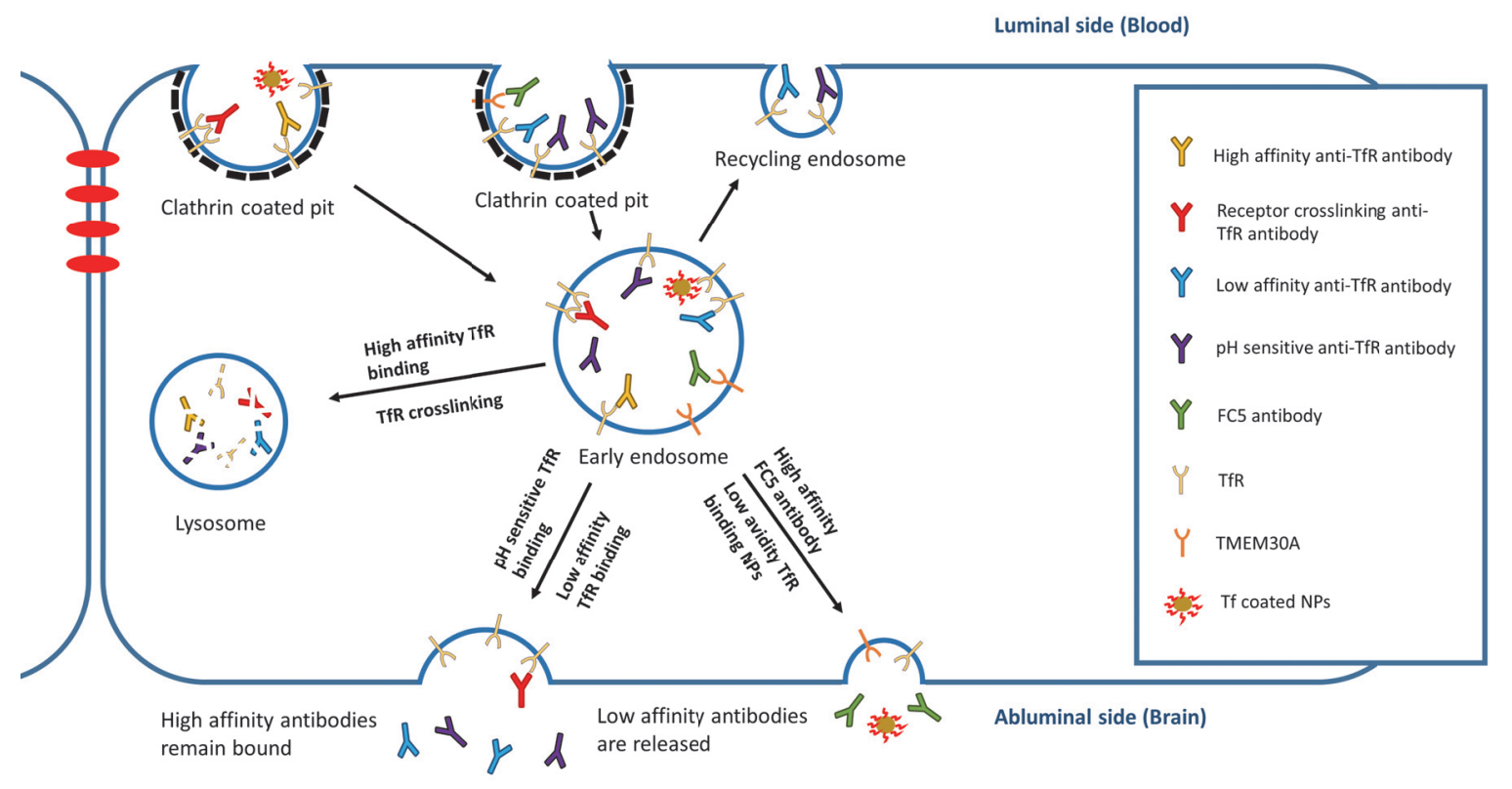

Figure 2. A schematic depiction of the various engineering optimization strategies for increased transcytosis of antibodies and NPs. High affinity monovalent and bivalent anti-TfR antibodies internalize readily into the EE, but then direct the antibody-receptor complex towards lysosomal degradation, possibly by crosslinking the TfR and altering 
its intracellular trafficking. While high affinity monovalent anti-TfR antibodies can transcytose the BBB, they remain bound to the receptor on the abluminal side, limiting the dose to the brain. In contrast, low affinity anti-TfR antibodies decrease antibody-TfR sorting to the lysosome, and can either be recycled back to the luminal side, or are transcytosed to the abluminal side where they dissociate from TfR, leading to increased brain accumulation. Similarly, Tf coated nanoparticles show a higher transcytosis capability when lowering the $\mathrm{Tf}$ coating content, resulting in reduced avidity. Further, $\mathrm{pH}-$ sensitive TfR binding antibodies that can dissociate from TfR in the acidic EE lead to increased transcytosis compared to $\mathrm{pH}$-insensitive antibodies. In the case of the single domain antibody FC5, increased affinity towards the receptor leads to an increase in the amount of transcytosed antibody, highlighting the fact that vectors utilizing different trafficking machinery may require customized optimization. 